\title{
Teaching Trauma: A Model for Introducing Traumatic Materials in the Classroom
}

\author{
Jessica D. Cless \\ Briana S. Nelson Goff
}

\begin{abstract}
University courses in disciplines such as social work, family studies, humanities, and other areas often use classroom materials that contain traumatic material (Barlow \& Becker-Blease, 2012). While many recommendations based on trauma theory exist for instructors at the university level, these are often made in the context of clinical training programs, rather than at the undergraduate level across disciplines. Furthermore, no organized model exists to aid instructors in developing a trauma-informed pedagogy for teaching courses on traumatic stress, violence, and other topics that may pose a risk for secondary traumatic stress in the classroom (Kostouros, 2008). This paper seeks to bridge the gap between trauma theory and implementation of sensitive content in classrooms of higher education, and presents a model of trauma-informed teaching that was developed in the context of an undergraduate trauma studies program. Implications and future directions for research in the area of trauma-informed university classrooms are discussed.
\end{abstract}

Keywords: Trauma-informed; teaching; higher education

Since the events of September 11, 2001, interest in courses on trauma and traumatic stress has spiked at universities across the country (Smith, 2002). While directly learning about trauma often occurs at the graduate level and in clinical programs of study, many disciplines outside of social work, human services, and clinical training programs also interact with materials and curriculum that involve trauma (Barlow \& Becker-Blease, 2012). For example, courses related to humanities, literature, art, and journalism often make use of films, readings, video clips, and guest speaker lectures which contain elements of traumatic experiences that may or may not trigger the students' own personal experiences (Dufresne, 2004; Dworznik \& Grubb, 2007). College and university professors have shared a variety of reasons for incorporating this type of trauma-related material into their classrooms, such as to prepare students for real-life situations they may encounter in the workforce, increase understanding of sociopolitical issues, or give life to boring topics or stories (Kostouros, 2008). Using this material in a classroom to promote student learning and engagement may be positive; however, it is not without some risk. Persons interacting with traumatic material may be at risk for secondary traumatic stress, also sometimes referred to as vicarious traumatization (Kostouros, 2008).

While trauma-informed (Bath, 2008) may have become somewhat of a buzzword in recent culture, it represents a crucial piece of implementing prevention and intervention services for individuals and families. According to Fallot and Harris (2009), a culture of trauma-informed care is rooted in the values of safety, trustworthiness, choice, collaboration, and empowerment. While gaining an understanding of the effects of trauma

Jessica D. Cless, M.S., LMFT, is a Graduate Teaching Assistant, School of Family Studies and Human Services, College of Human Ecology, Kansas State University, Manhattan, KS 66506-1403. Briana S. Nelson Goff, PhD, is Professor,

Family Studies and Human Services, College of Human Ecology, Kansas State University, Manhattan, KS 66506-1403.

Copyright @ 2017 Authors, Vol. 18 No. 1 (Spring 2017), 25-38, DOI: 10.18060/21177

(cc) BY

This work is licensed under a Creative Commons Attribution 4.0 International License. 
may be a first step to creating a culture of trauma-informed care, it is also crucial to shift patterns of thinking and behaviors that embrace this understanding (Harris \& Fallot, 2001).

The term "trauma-informed" has been most commonly applied to settings such as medical and mental health as well as prevention and intervention programs; less has been done to develop trauma-informed practices in classroom settings, especially in higher education. Recent recommendations for creation of trauma-informed systems have included providing education regarding traumatic stress for all professionals who may work with children and families (Ko et al., 2008), seeking to expand trauma-informed practice from its previous focus on mental health and medical practitioners. The current proposed model for trauma-informed teaching aims to begin to address the current gap for implementing trauma-informed teaching practices in higher education.

\section{Literature Review}

\section{Student Exposure to Trauma}

Primary exposure to trauma and diagnoses of trauma and stressor related disorders (see American Psychiatric Association [APA], 2013) may increase the risk for students to experience adverse reactions to course materials in classrooms that introduce traumatic materials (Cunningham, 2004). Traumatic experiences have been shown to be prevalent in both the general population (Chapman et al., 2012; Finkelhor, Turner, Ormrod, \& Hamby, 2009; Kilpatrick et al., 2013; Lukaschek et al., 2013) as well as populations of undergraduate students (Bernat, Ronfeldt, Calhoun, \& Arias, 1998; Frazier et al., 2009). A recent study found approximately $85 \%$ of students reported having experienced at least one traumatic event in their lifetime and $21 \%$ of students reported having experienced a trauma while in college (Frazier et al., 2009). Furthermore, specific types of students may be especially at-risk for having been exposed to trauma, such as veteran populations (Ackerman, DiRamio, \& Mitchell, 2009) and female students (Read, Ouimette, White, Colder, \& Farrow, 2011).

Veteran students represent an important population of college students that may have a history of trauma exposure. One study found a diagnosis of PTSD in veteran college students of OIF and OEF was associated with more problem drinking and physical aggression with others (Widome et al., 2011). Active duty and veteran service member students are more likely to have symptoms of posttraumatic stress than civilian students (Barry, Whiteman, \& MacDermid Wadsworth, 2012), furthering the need for traumainformed classroom practices. Recommendations have been made to universities regarding veteran student care and support, such as building awareness of veteran issues and creating external partnerships with military organizations (Rumann \& Hamrick, 2009) and creating a veteran-friendly climate through creating student veteran organizations and campus offices meant to address the needs of veterans (O'Herrin, 2011). These recommendations are meant to address the veteran student population as a whole on college campuses, however specific university faculty should also recognize how course content may affect veterans in the classroom. 


\section{Risk and Protective Factors}

While exposure to trauma should not be a barrier to students participating in education that includes trauma-related materials, in consideration of the high prevalence of trauma exposure, instructors should be mindful in introducing these materials in a classroom setting. It may also be helpful for instructors to be aware of risk and protective factors that may mitigate risk of reactivity after exposure to trauma has occurred. Trauma-exposure does not equate to traumatization (Bonanno, 2005) or a certain diagnosis of PTSD (Yehuda \& Flory, 2007); however, those who qualify for a PTSD diagnosis can be seen as more "reactive" or affected by the trauma. According to a 2000 meta-analysis, risk factors for PTSD include female gender, minority race, younger age, lower levels of education, previous trauma history, and childhood adversity (Brewin, Andrews, \& Valentine, 2000). Certain personality traits, such as neuroticism, have also been shown to increase risk for PTSD (Lauterbach \& Vrana, 2001). Factors such as social support, optimism, cognitive flexibility, and active coping such as problem-solving and learning to face fears have been identified as protective against traumatic stress (Haglund, Cooper, Southwick, \& Charney, 2007; Schumm, Briggs-Phillips, \& Hobfoll, 2006). Severity of the trauma along with a combination of risk and protective factors contribute to individual reactivity and adjustment, which may lend a more contextual understanding of student risk after exposure to trauma.

\section{Student Risk for Reactivity}

Various levels of risk may exist in regards to student reactions to course content. Students who have been exposed to some trauma but are not experiencing any distress are presumably at the lowest level of risk. The next level of risk may be comprised of those students experiencing some trauma-related symptoms that lead to discomfort or disrupt functioning. Finally, students at the highest level of risk would be those with a current diagnosis of posttraumatic stress disorder. Framing student levels of risk in this way can be helpful to instructors as they consider the need for thoughtful intervention of traumarelated materials in the classroom.

While it is important to consider student risk for reactivity based upon the students' own past experiences, it should be noted that reactions to course content may occur regardless of trauma history. Secondary traumatic stress (STS), which can occur when a person is not primarily experiencing a trauma themselves, but is exposed to traumatic material by hearing an account of another's trauma and as a result experiences a set of symptoms that mimic PTSD (APA, 2013) or acute stress disorder (Bober \& Regehr, 2005). In recognition of STS, instructors of courses on trauma and traumatic stress should introduce course content that contains traumatic material through responsible and strategic methods in order to minimize student risk for traumatization or secondary traumatic stress.

\section{Existing Trauma-Informed Teaching Recommendations}

In order to reduce occurrences of secondary traumatic stress in classroom settings, it has been recommended that university faculty be purposeful and cautious with the use of traumatic materials in the classroom (Kostouros, 2008). Specific biobehavioral, affective, 
cognitive, relational, and spiritual strategies may be utilized by students to protect against secondary traumatic stress, ranging from encouraging students to take time for recreation to being aware of their own maladaptive coping strategies (O'Halloran \& O'Halloran, 2001). For educators, Carello and Butler (2014) proposed steps for educators to make their educational practices more trauma-informed to include the importance of acknowledging the prevalence of students' trauma histories and making learning and safety central to the classroom experience. McCammon (1995) also provided suggestions to faculty for implementation of traumatic material to include establishing safety in the classroom, letting students know what materials are to be covered, carefully selecting examples, being knowledgeable of community and university referrals available to students, and handling student disclosures with care.

The International Society for Traumatic Stress Studies (ISTSS) provides best practice parameters for professionals in the areas of clinical work, research, and education. According to these parameters, educators should: communicate the potential impact of working in the field of trauma, such as vicarious traumatization; prepare students when materials may possibly be triggering; and avoid requiring assignments that encourage selfdisclosure without providing a genuine alternative (ISTSS, 2016). These best practice parameters are not stated to pertain to any specific academic or training setting. While these recommendations do align with trauma theory, many of them are largely based in clinical training populations and do not specifically address undergraduate students or academic programs across multiple disciplines. Furthermore, these recommendations are mainly individual suggestions for strategies on how to cope with course content, rather than representative of an organized model for implementation of content. The current paper attempts to bridge this gap between trauma theory and implementation of content in the classroom, through a model that was developed in the context of a trauma studies undergraduate program.

\section{Trauma-Informed Classroom Care Model}

The pedagogical need for introducing traumatic materials thoughtfully in the classroom arose over years of instruction and coordination of an undergraduate trauma studies program. This program, in which students are required to take courses on trauma and conflict, and may also choose elective courses related to trauma in topic areas such as violence, grief and loss, and life crises, was the context for the development of the TraumaInformed Classroom Care Model. This model is designed to aid instructors in recognizing and responding to student reactivity to traumatic materials in the classroom, with implications for instructional design. The following sections outline and describe each component of the model, with an emphasis on the research or evidence that was used to support the pieces of the model that have been combined to work together.

\section{Trauma Exposure}

Students may begin participating in courses that contain elements of trauma already having been exposed to trauma via their own life experiences, while other students without significant trauma histories may learn about the nature of trauma and traumatic stress for the first time in the classroom environment. In either case, exposure to elements of trauma 
is inevitable as course objectives may be to explore the nature of trauma, posttraumatic stress disorder, as well as common symptoms and reactions to stressful and/or traumatic events (Nelson Goff, 2016). As cited previously, university students may be exposed to traumatic experiences prior to and/or during their time in college (Frazier et al., 2009), with veteran students who may experience PTS symptoms or are diagnosed with PTSD (Barry et al., 2012). While instructors may or may not be aware of students' previous exposure to trauma, it can be assumed that many students will have had these experiences based upon general knowledge of trauma exposure. Some instructors may wish to help students evaluate how their own experiences may affect their experience in the course. This can be accomplished using a variety of methods, including assigning a reflective writing assignment at the beginning of the course in which students are prompted to consider how their personal background may "show up" in the course, and identifying which personal strengths may aid their learning experience.

\section{Reactions to Trauma}

Trauma exposure does not imply traumatization will occur, as many persons are resilient in the face of trauma and may not experience significant adverse reactions (Bonanno, 2005; Yehuda \& Flory, 2007). For this reason, trauma exposure and reactions are separate in the model. As possible previous trauma exposure may exist in students' own histories and re-exposure to trauma through the course materials will inevitably happen in the courses that are of current discussion, student reactivity to course materials may occur at any given point and time during the class. Student reactivity to traumatic materials is conceptualized using the Triphasic Model (Herman, 1997). This model describes three phases of trauma recovery: safety, remembrance and mourning, and reconnection. The three phases of trauma recovery in this model can serve as a way to understand a student's own level of reactivity to trauma, both personal and in the classroom.

Safety. During this stage of trauma recovery, survivors of trauma often feel unsafe in their own bodies, in their minds, in their relationships with others, or a combination of these. This phase may last weeks, months, or even years, depending on the severity of trauma (Herman, 1997). In the classroom setting, those in the safety stage may feel intense physiological reactions to lectures, videos, or other activities. Others may distance themselves from triggering materials by disengaging purposely or unintentionally. Of course these reactions, if they are present, may be easily masked in the modern classroom in which total student engagement is often interrupted by other students or means of technology (e.g. students using electronic devices during class for activities not related to the course, use of mobile phones or tablets). The instructor, then, should be aware of these reactions and open his/her mind to the possible various meanings of student overt reactivity or disengagement. Furthermore, the instructor should strive to make the classroom as safe and comfortable as possible.

Establishing safety has been noted as an important and often primary element of trauma-informed care (Bath, 2008; Fallot \& Harris, 2009). Safety can be fostered in the classroom in a number of ways, both structurally and through the instructor's use of self. First, making clear the course structure and content can help to establish a safe environment, which may be accomplished by making students aware of what content will 
be covered, communicating about when certain topics will be discussed, and clarifying the expectations of student participation in class. Furthermore, instructors should communicate to students clearly and often the acknowledgment of the intensity of the course materials, as well as being available to students. This need not be a long or overstated message, but it is important that the students are able to see the professor as a resource and as a safe person in the environment of a course that teaches trauma-related materials. In addition to verbal communication, instructors may also wish to include written notices on the course syllabus regarding possible student reactivity as well as resources available to students.

Remembrance and mourning. During this stage, survivors of trauma recall and work through their own memories of personal trauma, often reconstructing the trauma story (Herman, 1997). For survivors in this stage, the foundation of safety reached in the previous stage remains crucial to support healing during this time of recalling and examining personal trauma. While students in this stage may be less overtly or physically reactive to course materials, they may also be more emotionally or cognitively connected to the story of their own experience. Students may recount their own memories to instructors either verbally during class or through writing for course assignments, which warrants validation and monitoring on the part of the instructor as well as possible referrals and documentation of student interactions according to relevant university policies. For some students, learning about trauma in the classroom setting may lend new ideas and perspectives to their own processes of reconstruction or meaning making. For example, students learning for the first time about normal physiological reactions to threatening situations may begin to reframe actions taken in their own lives as protective rather than unresponsive or weak.

To support students who may be in this stage of recovery, instructors may wish to refrain from taking hard stances on what is "right" or "wrong" in relation to trauma reactions, treatment, or prevention, as it may disrupt a recovering student's own personal process. Mourning may increase reactivity and challenge students' beliefs and worldview, as they attempt to develop a cohesive understanding of their previous trauma experiences and work through the reactions that are a part of this stage of healing. The classroom should be supportive of this process, and instructors can take advantage of an open learning environment that is conducive to sharing new perspectives, and stories, which may help healing students to psychologically make room for new perspectives of their own trauma. Keeping with the values of choice and empowerment necessary in trauma-informed care (Fallot \& Harris, 2009), instructors should strive to allow students to move at their own pace and determine their own meanings about personal experiences or examples which may be present course content. While instructors should be supportive of students in this stage, they should also be mindful to stay within the scope of their teaching role. This may be particularly important for those instructors who work as social workers or clinicians in other settings, as separating these two roles may be more difficult.

Reconnection. The third stage of the Triphasic Model is that in which the survivor of trauma must create and define a new future self, as the past self was mourned in the previous stage. Often survivors in this stage are rediscovering a sense of "normalcy" in areas of their life that was altered by the trauma itself. According to Herman (1997), "the traumatized person recognizes that [he/she] has been a victim and understands the effects of [his/her] victimization. Now [he/she] is ready to incorporate the lessons of her traumatic 
experience into [his/her] life" (p. 197). Students in this stage may be comfortable disclosing their own experiences to the instructor or even to the whole class, and they may articulate integration of the trauma into their own lives. While students in this final stage of healing may present as less reactive to course content, use of this model may give instructors a clearer understanding as to how and why these students engage with the course materials, with peers, and with the instructor. The trauma-informed care value of empowerment (Fallot \& Harris, 2009) may be present in this stage as students come face-to-face with reminders of their journey and are able to retain a sense of self, power, and meaning. Even with students who have made significant progress in their healing journey, it is important to recognize these students in relationship to the course and how trauma-related materials may affect them.

\section{Student Disclosure of Trauma}

Students recovering from trauma in all three stages may disclose personal experiences of trauma as noted above. Student disclosure may occur in a variety of settings: in an individual meeting with the instructor, during on-campus discussions, or in writing through online message boards or in projects, papers, or other course assignments. Instructors should handle student disclosures with care, especially if shared in the larger classroom setting. If assignments that may prompt disclosure of personal history are required, such as reflective essays on personal stories, instructors should communicate with students about confidentiality and its possible limits, in compliance with the policies of their institution. Instructors may choose to refer students to on-campus counseling services who disclose recent experiences of trauma, as well as those experiences that may still affect their functioning. If students disclose information that suggests they may harm themselves or others, instructors should be knowledgeable about his or her school's process for reporting a student of concern to units such as student life or counseling services to promote student and community well-being.

\section{Flexibility}

In using the Trauma-Informed Classroom Care model, flexibility should be parallel to student levels of reactivity. This is meant to demonstrate students' varying needs in the classroom according to their own levels of reactivity to course content. The model posits that students with higher levels of reactivity to traumatic materials should be met with higher levels of flexibility within the course. While many students may be exposed to these topics for the first time by means of the course content, other students will have previously been exposed to trauma and may or may not be triggered by materials in the class. Reactions to trauma are variant, and many individuals show signs of resilience even in the face of trauma (Bonanno, 2005), making it difficult to predict which students may be triggered during the course, even if information about a student's previous trauma exposure is known by the instructor.

Flexibility may vary in each course according to the needs of each class and instructor. Some recommendations for implementing flexibility in a course setting include allowing various options of assignments with a mix of objective and subjective choices, allowing students to attend a specific lecture remotely, or offering alternative ways for students to 
comfortably demonstrate learning. This can be accomplished through offering alternative assignments, allowing students a certain number of absences, or having an incomplete policy in place for students who may need to stop the course and retake it in the future. It should be noted that flexibility in a course does not mean a lack of structure or allowing students to do whatever they would like to do or not meet academic requirements. Instructors should be able to allow certain flexibilities in the course, while maintaining clear structure and boundaries.

\section{Course Progression}

Especially in courses in which traumatic topics are the core focus of the class, it is expected that student reactions to the course may vary over time as topics are introduced. For example, a general course on identifying trauma and traumatic stress may heavily focus on sexual assault at one point in the course, but move to other topics as the course progresses. This is important to recognize as student personal histories with exposure to specific traumas are unknown to instructors, and reactions to more specific content within a course may occur at various points over the semester. For this reason, instructors should be cognizant of student reactions and be available to students throughout the course.

Communication with students directly regarding course progression can be a tool for instructors to utilize in order to promote student well-being in the course. In sending electronic notifications or emails to the class, instructors can acknowledge the intensity of the course material and offer to meet with students who may be experiencing distress. These statements can also be added the course syllabus, as a way to display this message throughout the duration of the course. Instructors can also make an effort to check in with students who seem to have withdrawn from the course by not attending or completing assignments. These moments of communication may seem insignificant, but they can be strategically implemented in order to create a safe and supportive environment for students exposed to traumatic materials in the course content.

\section{Assessment}

In courses with a heavy focus on trauma related topics, assessment should always be part of the course. Whereas courses that do not use traumatic materials may assess student progress toward learning outcomes, this process is of even greater importance in courses with traumatic materials not only to assess student learning but also to monitor student reactivity. Assessment of student learning outcomes often comes in the form of an assignment (e.g., test, quiz, essay) while assessing student reactivity may take place on various levels. While some students may feel comfortable approaching instructors to share any adverse reactions to course materials, other students may withdraw from the course in various ways. Student withdrawal may include poor or no attendance, low or no participation in course assignments and discussions, or failing to respond to assignments in-depth. Instructors should also be aware of more overt reactions to the course content (such as tearfulness or anger) and be ready to respond accordingly. As previously mentioned, students who disclose having personal histories that may increase risk for reactivity to course content should be offered referrals to university and/or community resources that he or she may choose to utilize as a source of support and method of coping. 
If students reach a higher level of concern, exhibited by making statements that would be indicative of harm to self or others, instructors should be ready to make a report without the student's consent in order to ensure the student's well-being and safety. Although this can be difficult for instructors, as they may fear it could jeopardize the rapport they have built with their student(s), student well-being is of primary concern and reporting to academic or student services administrators allows a broader network of care and support to be developed for the student.

\section{Discussion and Future Directions}

This paper has proposed a trauma-informed teaching model for use in university courses that cover trauma and traumatic stress, violence, sexual assault, or other courses that may be completely or partially focused on topics that contain elements of trauma. Building on the work of other scholars who have addressed this topic, this model was constructed using a well-known trauma treatment model as a foundation for understanding reactivity to trauma, and expanding it to be relevant for the classroom. This work contributes to existing trauma-informed recommendations for teaching by providing a framework through which the need for these recommendations can be understood. Using a trauma-informed model for teaching should not be the only step to becoming "traumainformed." Instructors of university courses should take additional steps in order to respond to the varying needs of their students. Educating students about and communicating the need for self-care in courses that incorporate trauma-related content may encourage prevention of burnout and secondary trauma in students (O'Halloran \& O'Halloran, 2001). Steps such as providing trainings to help instructors identify students at risk for mental health problems, which are prevalent among college students (Hunt \& Eisenberg, 2010), implementing mentoring programs (Santos \& Reigadas, 2005), and making clear to both instructors and students campus resources for mental health, advocacy, and advising are also recommended for holistic care of students in addition to mindful introduction of sensitive materials in the classroom.

This model may help those in the social work profession to more clearly implement the competencies outlined by the Council on Social Work Education (CSWE). Specifically, Competency \#3, which calls for social workers to understand and promote the advancement of human rights and social, economic, and environmental justice, to include safety and education (CSWE, 2015). The creation of this model was founded on the recognition of the widespread experience of trauma, and aligns with the CSWE's call for professionals to "engage in practices that advance social, economic, and environmental justice" (CSWE, 2015 , p. 8). Instructors at the university level should be a part of this process, and can both model and practice social justice with students in the classroom. Furthermore, the CSWE regards dignity and worth of the person as a central professional value in the field of social work that should be fostered in the educational environment (CSWE, 2015). Since this proposed model centers on understanding the student's own level of reactivity and strives to help instructors respond accordingly to foster student well-being, the values of dignity and worth are exemplified through its use.

It should be acknowledged that this model was developed in the context of a nonclinical trauma studies program with courses that focus on topics such as trauma and 
violence directly. In this way, the model has been formulated through the experience of teaching about trauma and implementing traumatic materials into the classroom due to the general focus of the course itself. However, other instructors may find that their own students are having reactions to materials that are not overtly "triggering" or seemingly traumatic in content. The authors denote this as a difference between teaching trauma, in which traumatic materials are the focus of the course content (Carello \& Butler, 2014), and trauma-informed teaching, in which students with prior histories of trauma exposure may react adversely to any number of cues that are presented in a classroom not related stereotypically to trauma but personally meaningful to the student (Cunningham, 2004; Dufresne, 2004). This model may be flexible for both contexts; however, as the elements of trauma exposure and trauma re-exposure in the classroom setting may also be true for students taking courses unrelated to psychological trauma or distress. More development of the current model and empirical investigation of teaching trauma and trauma-informed teaching may help to clarify any existing differences in the needs of students with trauma histories in both types of classes.

It is important to remember that in conceptualizing student reactivity to traumatic course materials using Herman's (1997) Triphasic Model, instructors do not fill the role of helping professionals, such as social workers, psychologists, therapists, or doctors. All care toward students should be provided by licensed professionals. However, the Triphasic Model does provide a frame of reference that can inform instructors of signs and symptoms of student reactivity at various levels of the recovery process for students. Again, as it is expected that students will be present in the course with a history of trauma that may have occurred in the distant or recent past, or is currently ongoing, leaving students vulnerable to adverse reactions to course materials that should be thoughtfully implemented. Instructors may need to make referrals or work with student services offices offered at their university in order to ensure student care and safety.

While this pedagogical model recognizes the possibility for student reactivity to trauma-related course materials, it does not address how the histories and reactions of instructors may also influence the higher education classroom. Further study and conceptualization should be done to consider how this added element may affect the current model and trauma-informed teaching as a whole. Perhaps the greatest focus of attention in advancing knowledge related to trauma-informed teaching at the university-level is to provide empirical support for models such as these authors propose, as well as for traumainformed interventions. While in the past many recommendations have been made for teaching trauma, no studies to date have tested these recommendations. An empirically supported model that can be used as a lens in how to teach traumatic materials in the classroom will give university faculty the opportunity to more carefully introduce students to trauma in an academic setting, while keeping in mind the students' own levels of reactivity and steps to healing from previous trauma.

\section{References}

Ackerman, R., DiRamio, D., \& Mitchell, R. L. G. (2009). Transitions: Combat veterans as college students. New Directions for Student Services, 2009(126), 5-14. doi: https://doi.org/10.1002/ss.311 
American Psychiatric Association. (2013). Diagnostic and statistical manual of mental disorders: DSM-5. Washington, D.C: American Psychiatric Association. doi: https://doi.org/10.1176/appi.books.9780890425596

Barlow, M. R., \& Becker-Blease, K. (2012). Caring for our students in courses with potentially threatening content. Psychology of Women Quarterly, 36(2), 240-243. doi: https://doi.org/10.1177/0361684312442662

Barry, A. E., Whiteman, S. D., \& MacDermid Wadsworth, S. M. (2012). Implications of posttraumatic stress among military-affiliated and civilian students. Journal of American College Health, 60(8), 562-573. doi: https://doi.org/10.1080/07448481.2012.721427

Bath, H. (2008). The three pillars of trauma-informed care. Reclaiming Children and Youth, 17(3), 17-21. Retrieved from https://s3-us-west2.amazonaws.com/cxl/backup/prod/cxl/gklugiewicz/media/507188fa-30b7-8fd4aa5f-ca6bb629a442.pdf

Bernat, J. A., Ronfeldt, H. M., Calhoun, K. S., \& Arias, I. (1998). Prevalence of traumatic events and peritraumatic predictors of posttraumatic stress symptoms in a nonclinical sample of college students. Journal of Traumatic Stress, 11(4), 645-664. doi: https://doi.org/10.1023/A:1024485130934

Bober, T., \& Regehr, C. (2005). Strategies for reducing secondary or vicarious trauma: Do they work? Brief Treatment and Crisis Intervention, 6(1), 1-9. doi: https://doi.org/10.1093/brief-treatment/mhj001

Bonanno, G. A. (2005). Resilience in the face of potential trauma. Current Directions in Psychological Science, 14(3), 135-138. doi: https://doi.org/10.1111/j.09637214.2005.00347.x

Brewin, C. R., Andrews, B., \& Valentine, J. D. (2000). Meta-analysis of risk factors for posttraumatic stress disorder in trauma-exposed adults. Journal of Consulting and Clinical Psychology, 68(5), 748-766. doi: https://doi.org/10.1037/0022006X.68.5.748

Brown, L. S. (2004). Feminist paradigms of trauma treatment. Psychotherapy Research, Practice and Training, 41(4), 464-471. doi: https://doi.org/10.1037/0033$\underline{3204.41 .4 .464}$

Carello, J., \& Butler, L. D. (2014). Potentially perilous pedagogies: Teaching trauma is not the same as trauma-informed teaching. Journal of Trauma \& Dissociation, 15(4), 153-168. doi: https://doi.org/10.1080/15299732.2014.867571

Chapman, C., Mills, K., Slade, T., McFarlane, A. C., Bryant, R. A., Creamer, M., ... \& Teesson, M. (2012). Remission from post-traumatic stress disorder in the general population. Psychological Medicine, 42(8), 1695-1703. doi: https://doi.org/10.1017/S0033291711002856 
Council on Social Work Education. (2015). Educational policy and accreditation standards for baccalaureate and master's social work programs. Retrieved from http://www.cswe.org/File.aspx?id=81660

Cunningham, M. (2004). Teaching social workers about trauma: Reducing the risks of vicarious traumatization in the classroom. Journal of Social Work Education, 40(2), 305-317. Retrieved from http://www.tandfonline.com/doi/abs/10.1080/10437797.2004.10778495

Dufresne, M. (2004). Teaching about trauma. The Quill, 92(6), 29. Retrieved from https://www.highbeam.com/doc/1P3-709147541.html

Dworznik, G., \& Grubb, M. (2007). Preparing for the worst: Making a case for trauma training in the journalism classroom. Journalism \& Mass Communication Educator, 62(2), 190-210. doi: https://doi.org/10.1177/107769580706200206

Fallot, R. D., \& Harris, M. (2009). Creating cultures of trauma-informed care (CCTIC): A self-assessment and planning protocol. Retrieved from https://www.healthcare.uiowa.edu/icmh/documents/CCTICSelfAssessmentandPlanningProtocol0709.pdf

Finkelhor, D., Turner, H., Ormrod, R., \& Hamby, S. L. (2009). Violence, abuse, and crime exposure in a national sample of children and youth. Pediatrics, 124, 1-13. doi: https://doi.org/10.1542/peds.2009-0467

Frazier, P., Anders, S., Perera, S., Tomich, P., Tennen, H., Park, C., \& Tashiro, T. (2009). Traumatic events among undergraduate students: Prevalence and associated symptoms. Journal of Counseling Psychology, 56(3), 450-460. doi: https://doi.org/10.1037/a0016412

Haglund, M., Cooper, N., Southwick, S., \& Charney, D. (2007). 6 keys to resilience for PTSD and everyday stress. Current Psychiatry, 6(4), 23-30.

Harris, M., \& Fallot, R. D. (2001). Envisioning a trauma-informed service system: A vital paradigm shift. New Directions for Mental Health Services, 2001(89), 3-22. doi: https://doi.org/10.1002/yd.23320018903

Herman, J. (1997). Trauma and recovery. New York: Basic Books.

Hunt, J. H., \& Eisenberg, D. (2010). Mental health problems and help-seeking behavior among college students. Journal of Adolescent Health, 46(1), 3-10. doi: https://doi.org/10.1016/j.jadohealth.2009.08.008

International Society for Trauma and Traumatic Stress [ISTSS]. (2016). ISTSS Best Practice Parameters. Retrieved from https://www.istss.org/ISTSS_Main/media/Documents/ISTSS_Best_Practice_Paramet ers1.pdf

Kilpatrick, D. G., Resnick, H. S., Milanak, M. E., Miller, M. W., Keyes, K. M., \& Friedman, M. J. (2013). National estimates of exposure to traumatic events and 
PTSD prevalence using DSM-IV and DSM-5 criteria. Journal of Traumatic Stress, 26(5), 537-547. doi: https://doi.org/10.1002/jts.21848

Ko, S. J., Ford, J. D., Kassam-Adams, N., Berkowitz, S. J., Wilson, C., Wong, M., Brymer, M. J., \& Layne, C. M. (2008). Creating trauma-informed systems: Child welfare, education, first responders, health care, juvenile justice. Professional Psychology: Research and Practice, 39(4), 396-404. doi: https://doi.org/10.1037/0735-7028.39.4.396

Kostouros, P. (2008). Using traumatic material in teaching. Transformative Dialogues: Teaching \& Learning Journal, 2(1), 1-7. Retrieved from http://www.kpu.ca/sites/default/files/Teaching\%20and\%20Learning/TD.2.1_Kostour os_Using_Traumatic_Material_in_Teaching.pdf

Lauterbach, D., \& Vrana, S. (2001). The relationship among personality variables, exposure to traumatic events, and severity of posttraumatic stress symptoms. Journal of Traumatic Stress, 14(1), 29-45. doi: https://doi.org/10.1023/A:1007831430706

Lukaschek, K., Kruse, J., Emeny, R. T., Lacruz, M. E., von Eisenhart Rothe, A., \& Ladwig, K. H. (2013). Lifetime traumatic experiences and their impact on PTSD: A general population study. Social Psychiatry and Psychiatric Epidemiology, 48(4), 525-532. doi: https://doi.org/10.1007/s00127-012-0585-7

McCammon, S. L. (1995). Painful pedagogy: Teaching about trauma in academic and training settings. In B. H. Stamm (Ed.), Secondary traumatic stress: Self-care issues for clinicians, researchers, and educators. Lutherville, MD: Sidran Press.

Nelson Goff, B. S. (2016). Understanding trauma and traumatic stress [Syllabus]. Manhattan, KS: Kansas State University.

O'Halloran, S. M., \& O'Halloran, T. (2001). Secondary traumatic stress in the classroom: Ameliorating stress in graduate students. Teaching of Psychology, 28(2), 92-97. doi: https://doi.org/10.1207/S15328023TOP2802_03

O’Herrin, E. (2011). Enhancing veteran success in higher education. Peer Review, 13(1), 15-18. Retrieved from Retrieved from http://go.galegroup.com.er.lib.kstate.edu/ps/i.do?id=GALE\%7CA273081363\&v=2.1\&u=ksu\&it=r\&p=AONE\&sw= w\&asid=63ec2aff80d93b6945b32f3d085b2134

Read, J. P., Ouimette, P., White, J., Colder, C., \& Farrow, S. (2011). Rates of DSM-IVTR trauma exposure and posttraumatic stress disorder among newly matriculated college students. Psychological Trauma, 3(2), 148-156. doi: https://doi.org/10.1037/a0021260

Rumann, C. B., \& Hamrick, F. A. (2009). Supporting student veterans in transition. New Directions for Student Services, 2009(126), 25-34. doi: https://doi.org/10.1002/ss.313

Santos, S. J., \& Reigadas, E. T. (2005). Understanding the student-faculty mentoring process: Its effects on at-risk university students. Journal of College Student Retention, 6(3), 337-357. doi: https://doi.org/10.2190/KGVC-7218-DPER-RMBC 
Schumm, J. A., Briggs-Phillips, M., \& Hobfoll, S. E. (2006). Cumulative interpersonal traumas and social support as risk and resiliency factors in predicting PTSD and depression among inner-city women. Journal of Traumatic Stress, 19(6), 825-836. doi: https://doi.org/10.1002/jts.20159

Smith, D. (2002). Undergrads flock to classes on trauma. Monitor on Psychology, 33(4), 34. Retrieved from http://www.apa.org/monitor/apr02/undergrads.aspx

Widome, R., Kehle, S. M., Carlson, K. F., Laska, M. N., Gulden, A., \& Lust, K. (2011). Post traumatic stress disorder (PTSD) and health risk behaviors among Afghanistan and Iraq war veterans attending college. American Journal of Health Behavior, 35(4), 387-392. doi: https://doi.org/10.5993/AJHB.35.4.1

Yehuda, R., \& Flory, J. D. (2007). Differentiating biological correlates of risk, PTSD, and resilience following trauma exposure. Journal of Traumatic Stress, 20(4), 435447. doi: https://doi.org/10.1002/jts.20260

Author note: Address correspondence to: Jessica Cless, College of Human Ecology, Kansas State University, Manhattan, KS 66506-1403. Email jhigh@ksu.edu 